\title{
Infiltrating bone marrow mesenchymal stem cells (BM-MSCs) increase prostate cancer cell invasion via altering the CCL5/ HIF2a/androgen receptor signals
}

\author{
Jie Luo ${ }^{1,2,3,4,5}$, Soo Ok Lee ${ }^{1,2,3,4,5}$, Yun Cui ${ }^{1,2,3,4,5}$, Rachel Yang ${ }^{1,2,3,4,5}$, Lei Li $i^{1,2,3,4,5,6}$, \\ Chawnshang Chang ${ }^{1,2,3,4,5,7}$ \\ ${ }^{1}$ Department of Pathology, University of Rochester, Rochester, NY 14642, USA \\ ${ }^{2}$ Department of Urology, University of Rochester, Rochester, NY 14642, USA \\ ${ }^{3}$ Department of Radiation Oncology, University of Rochester, Rochester, NY 14642, USA \\ ${ }^{4}$ Department of Biology, University of Rochester, Rochester, NY 14642, USA \\ ${ }^{5}$ The Wilmot Cancer Center, University of Rochester, Rochester, NY 14642, USA \\ ${ }^{6}$ Sex Hormone Research Center, Department of Urology, The First Affiliated Hospital, Xian Jiaotong University, Xian 710061, China \\ ${ }^{7}$ Sex Hormone Research Center, China Medical University and Hospital, Taichung, 404, Taiwan \\ Correspondence to: \\ Chawnshang Chang, e-mail: chang@urmc.rochester.edu \\ Lei Li, e-mail: lilydr@163.com \\ Keywords: CCL5, bone marrow mesenchymal stem cells, HIF2a, androgen receptor, prostate cancer \\ Received: February 26, $2015 \quad$ Accepted: July 17, $2015 \quad$ Published: July 27, 2015
}

\section{ABSTRACT}

Several infiltrating cells in the tumor microenvironment could influence the cancer progression via secreting various cytokines. Here, we found the CCL5 secreted from BM-MSCs suppressed androgen receptor (AR) signals via enhancing the expression of hypoxia inducible factor $2 a(H I F 2 a)$ in prostate cancer (PCa) cells. Mechanism dissection revealed that the increased HIF2a might alter the AR-HSP9O interaction to suppress the AR transactivation, and inhibition of HIF2a reversed the BM-MSCsincreased PCa stem cell population and PCa cells invasion. Importantly, CCL5 could suppress the prolyl hydroxylases (PHDs) expression, which might then lead to suppress VHL-mediated HIF2a ubiquitination. Together, these results demonstrated that the CCL5 signals from infiltrating BM-MSC cells to HIF2a signals within PCa cells might play a key role to increase PCa stem cell population and PCa metastasis via altering the AR signals. Targeting this newly identified CCL5/HIF2a/AR axis signal axis may allow us to develop a novel way to suppress PCa metastasis.

\section{INTRODUCTION}

Recent studies suggested that several cell types in the prostate tumor microenvironment (TME) might contribute to the prostate cancer $(\mathrm{PCa})$ progression [1-11]. For example, infiltrating macrophages might promote PCa metastasis via modulation of CCL2/CCR2STAT3 signaling [7-9] and recruited endothelial cells might also be able to promote $\mathrm{PCa}$ metastasis via modulation of IL6 signaling [10]. Our previous study demonstrated that infiltrating bone marrow derived mesenchymal stem cells (BM-MSCs) might be able to enhance PCa cell invasion via altering the cancer stem cell differentiation. The mechanism dissection revealed that this regulation involves the modulation of CCL5 and AR signaling [11]. The CCL5 secreted from BM-MSCs can increase the cancer stem cell and EMT markers, such as the CD133, ZEB-1 and CXCR4.

The findings that $\mathrm{AR}$ in individual cells within the TME might play differential roles (positive $v s$ negative roles) could further complicate the androgen/AR signaling in PCa progression [7-13] and raised special questions about the current androgen deprivation therapy (ADT), which systematically suppresses/reduces androgen from binding to $\mathrm{AR}$ in every cell, to suppress the progression of $\mathrm{PCa}$, a disease that has become the most prevalent cancer among males in United States with the 2nd highest mortality rate. [8, 9, 14, 15]. Therefore, better understanding the differential AR signaling in each cell within the TME and from those distinct AR signals to develop better target(s) to modulate AR-mediated PCa in selective cells may help us to battle PCa in future. 
Hypoxia-inducible factor (HIF) is the central component in response to hypoxia in the cell. Substantial studies reveal that HIF is important for the tumor growth and metastasis [16]. Under hypoxia conditions, the HIF expression can be immediately regulated by the proteasome pathway. The prolyl hydroxylases (PHDs), members of the iron- and 2-oxoglutarate-dependent dioxygenase enzyme family, can be suppressed by hypoxia. The PHDs can hydroxylate the HIFs and then promote the ubiquitination of HIF through its E3 ligase-von Hippel-Lindau tumor suppressor (VHL). There are several genes down stream of HIFs that play an important role in the cancer progression including VEGF [17]. A recent study revealed that the HIFs also regulate the cancer stem cell population [18].

Here we identify that HIF2 $\alpha$ [19-21] may link the signaling between CCL5 and AR to enable the recruited BMMSCs to promote PCa cell invasion. Mechanism dissection found CCL5 might function through modulation of HIF $2 \alpha$ ubiquitination to stabilize the HIF $2 \alpha$ protein, and then alter the interaction of HSP90 and AR that resulted in suppression of AR nuclear translocation and AR transactivation.

\section{RESULTS}

\section{BM-MSCs Increase PCa stem cell population and PCa cell invasion via enhancing HIF2a expression}

We investigated whether the stem cell population in parental PCa cells can be altered after co-cultured with BM-MSCs in a co-culture system as shown in Figure 1a. We observed significant increase in PCa stem cell population when C4-2 cells were co-cultured with BMMSCs, compared to non-co-cultured condition via sphere formation assay (Figure 1b) [22]. We further examined whether this increased stem cell population could influence the invasion ability of PCa cells [23], and results revealed that the invasion ability of these PCa cells are significantly enhanced (Figure 1c). These results confirm our previous report showing the recruited BM-MSCs into $\mathrm{PCa}$ led to increase the stem cell population and invasion ability of PCa cells [11].

To dissect the molecular mechanism(s) by which BM-MSCs increase PCa stem cell population, we examined whether BM-MSCs increase stem cell population through activation of several potential signaling pathways that have been reported to be involved in expansion of cancer stem cell population. Among several candidates, we were interested in HIF2 $\alpha$ signaling as early studies suggested that HIF $2 \alpha$ could induce tumor aggressiveness and expand the cancer stem cell population [24, 25].

We then tested the expression of HIF $2 \alpha$ in LNCaP and C4-2 cells after co-culture with BM-MSCs (Figure 1d), and found the increased HIF2 $\alpha$ at protein (Figure 1d) and not at mRNA levels (Figure 1e) in these two PCa cells when co-cultured with BM-MSCs.
Similar result were also obtained when we orthotopically xenografted CWR22RV1 PCa cells with BMMSCs into mouse prostate showing increasing HIF2 $\alpha$ expression (Figure 1f).

Together, results from in vitro cell lines and in vivo mice studies suggest that BM-MSCs may contribute to the increase of the HIF $2 \alpha$ expression in PCa cells.

To further examine the influences of increased HIF $2 \alpha$ on the BM-MSCs-increased PCa stem cell numbers and $\mathrm{PCa}$ cell invasion, we applied the interruption approach via knocking-down the HIF2 $\alpha$ in PCa cells, and results revealed that knocking-down HIF $2 \alpha$ (Figure $2 \mathrm{a}$ ) resulted in suppression of BM-MSCs-enhanced C4-2 stem cell population (Figure 2b) and consequent $\mathrm{C} 4-2$ cell invasion (Figure 2c). Importantly, we found knocking-down HIF2 $\alpha$ also suppressed those increased stem cell markers (CD133) and metastasis related genes, ZEB-1 and CXCR4 [11, 26] (Figure 2d).

Taken together, results from Figure 1 and 2 suggest that HIF2 $\alpha$ may play a key role in mediating the BM-MSCs co-culture effects on the increase of PCa stem cell numbers and PCa cell invasion ability.

\section{BM-MSCs secrete CCL5 to induce the HIF2 $\alpha$ expression}

Next we asked what signaling in BM-MSCs could influence the up-regulation of HIF $2 \alpha$ expression in PCa cells upon BM-MSCs co-culture. Since early studies indicated that CCL5 is the key cytokine that triggers PCa stem cell increase and PCa cell invasion [11], we were interested to see its impact on the HIF2 $\alpha$ expression. By adding the functional CCL5 recombinant protein (rCCL5) directly into the culture of various PCa cell lines, we found increased HIF2 $\alpha$ expression in PCa cells (Figure 3a), and blocking CCL5 (via CCL5 neutralizing antibody) suppressed the BM-MSCs induced HIF2 $\alpha$ expression (Figure $3 b$ ).

Together, results from Figure $3 a-3 b$ suggest that CCL5 secreted from BM-MSCs may play a key role in modulating the increased expression of HIF $2 \alpha$ in PCa cells.

Furthermore, knocking-down HIF $2 \alpha$ significantly attenuated the CCL5 effect in increasing PCa stem cell numbers (Figure 3c) and PCa cells invasion (Figure 3d). We also observed the knocking-down HIF $2 \alpha$ in PCa cells attenuated the CCL5 effect of up-regulation of CD133 and ZEB-1 (Figure 3e).

Together, results from Figure $3 c-3 e$ suggest that the CCL5 effect in enhancing PCa stem cell population and PCa cell invasion is via altering the HIF $2 \alpha$ signals.

\section{CCL5 suppresses HIF2 $\alpha$ ubiquitination by inhibiting the VHL-HIF2 $\alpha$ interaction}

To further dissect the molecular mechanism(s) by which CCL5 increases HIF2 $\alpha$, we investigated whether CCL5 enhances the HIF $2 \alpha$ mRNA expression. As shown in Figure $4 \mathrm{a}$, addition of rCCL5 into the PCa cell culture could 
a.

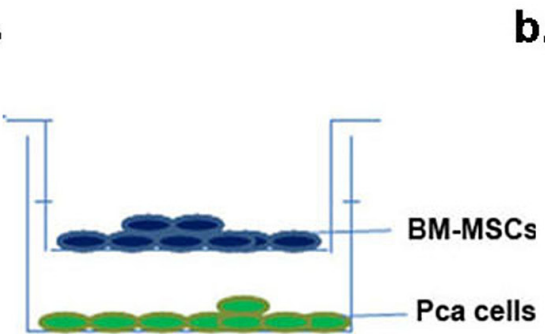

c.

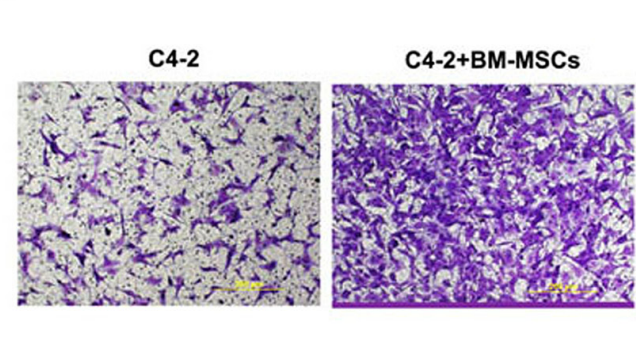

Q

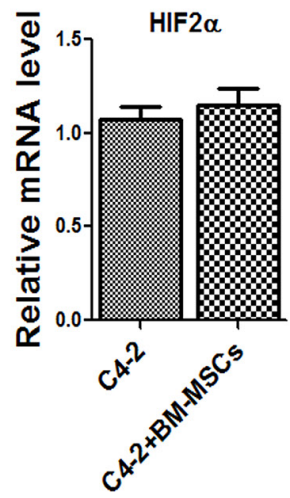

f. b.
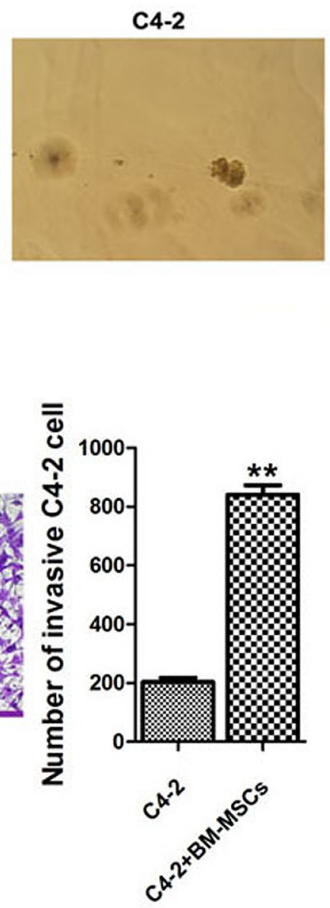

C4-2+BM-MSCs
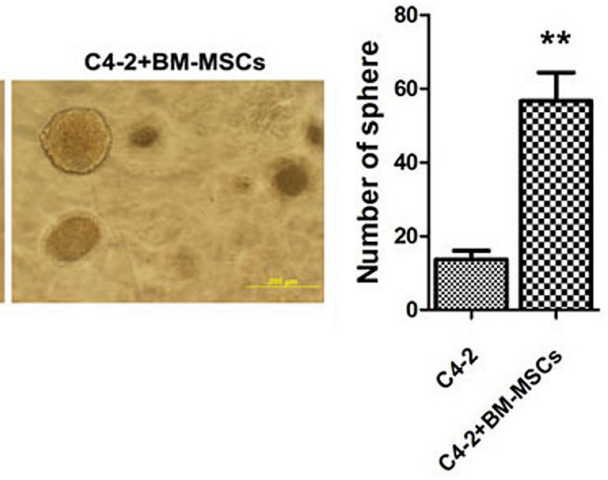

d.
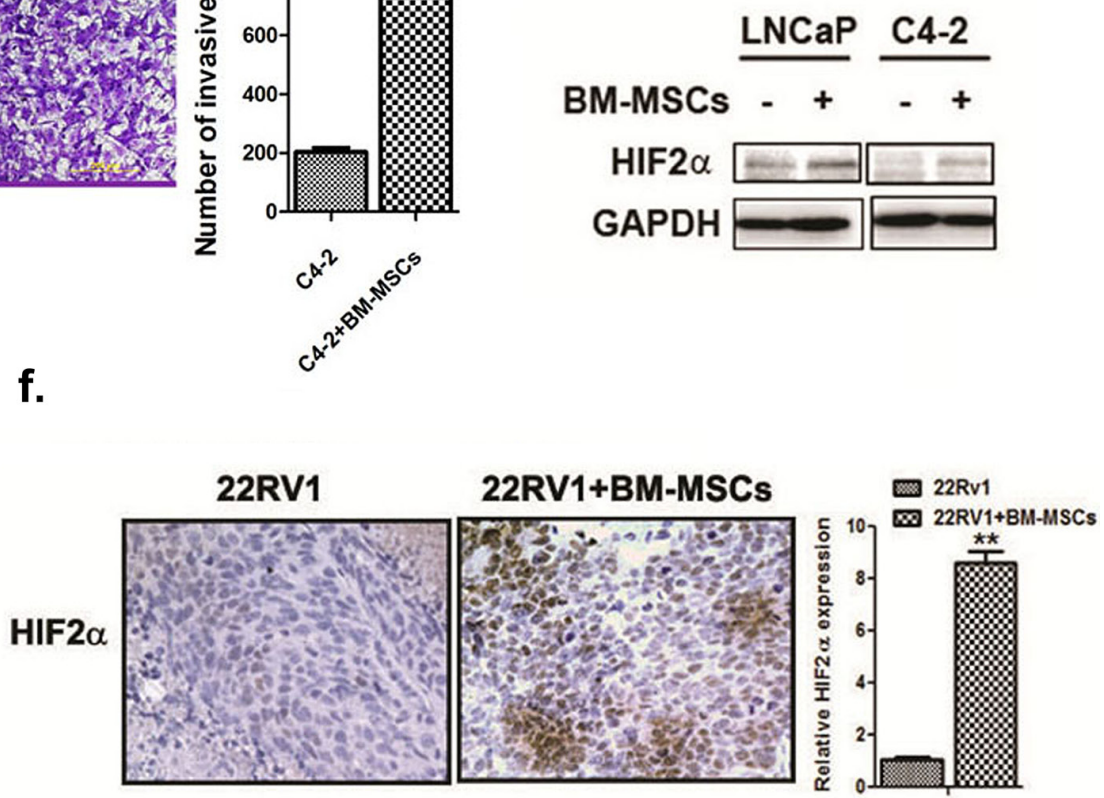

Figure 1: BM-MSCs increase HIF2 $\alpha$ expression in PCa cells. a. The cartoon demonstrating BM-MSCs and PCa cells co-culture $1 \times 10^{5}$ BM-MSCs (with media as control) were placed in upper chambers of the transwell plates ( $0.4 \mu \mathrm{m}$ membrane) while PCa cells $\left(1 \times 10^{6}\right)$ were placed in lower chambers. b. Sphere formation assay. The PCa cells were co-cultured with the primary MBM-MSCs (media used as control) in $0.4 \mu \mathrm{M}$ membrane transwell plates for 5 days. Cells were then mixed with Matrigel (1:1, v/v), plated in 24-well plates, and cultured for 10 days. Quantification was shown at right. c. Invasion assay result. The C4-2 cells $\left(1 \times 10^{5}\right)$ were co-cultured with the mouse primary BM-MSCs for 3 days in transwell plates $(0.4 \mu \mathrm{M}$ membrane). The invaded cells were stained by toluidine blue, and the positively stained cells were counted from 5 random areas. Quantitation was shown at right. d. Western blot analysis of HIF $2 \alpha$ expressions. The LNCaP and C4-2 cells were co-cultured with or without the primary mouse BM-MSCs and HIF2 $\alpha$ expression was analyzed. e. qPCR analysis of HIF $2 \alpha$ mRNA level in C4-2 cells with or without BM-MSCs co-culture. f. HIF2 $\alpha$ IHC staining of the tumor tissues obtained from the CWR22RV1 (22RV1) xenografted mice, with or without co-implantation with the primary BM-MSCs.

not increase the HIF2 $\alpha$ at mRNA level significantly. Next, we tested whether CCL5 could regulate the stability of HIF $2 \alpha$ protein. The C4-2 cells were treated with rCCL5, and the proteasome inhibitor MG132 or vehicle, and results revealed that MG132 blocked the CCL5-increased HIF2 $\alpha$ expression (Figure 4b), suggesting that the CCL5 secreted by BM-MSCs may affect the protein stability of HIF $2 \alpha$ in PCa cells.

We further investigated whether CCL5 can influence ubiquitination of HIF2 $\alpha$ and the co-immunoprecipitation (CoIP) assay revealed that the CCL5 strongly inhibited the interaction between ubiquitin (Ub) and HIF2 $\alpha$. This suggests that the CCL5 may suppress the ubquitination of HIF $2 \alpha$. (Figure 4c) Next, we examined the expression of PHDs, members of the iron- and 2-oxoglutarate-dependent dioxygenase enzyme family [27], in $\mathrm{PCa}$ cells upon BM-MSCs co-culture, as early studies suggested the PHDs can catalyze hydroxylation of HIF2 $\alpha$ and then promote its binding to VHL, a E3-ligase of the HIF2 $\alpha$ [28]. It can be speculated that the HIF $2 \alpha$ molecule may undergo degradation via proteasomal degradation after binding to VHL. Indeed, we found that expressions of the PHD1 and PHD4 molecules in PCa cells were dramatically decreased 
a.

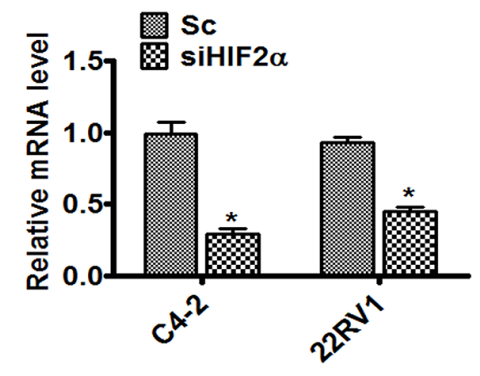

c.

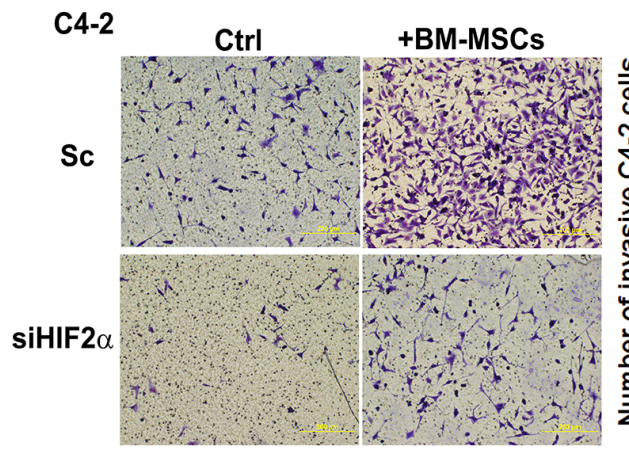

b.
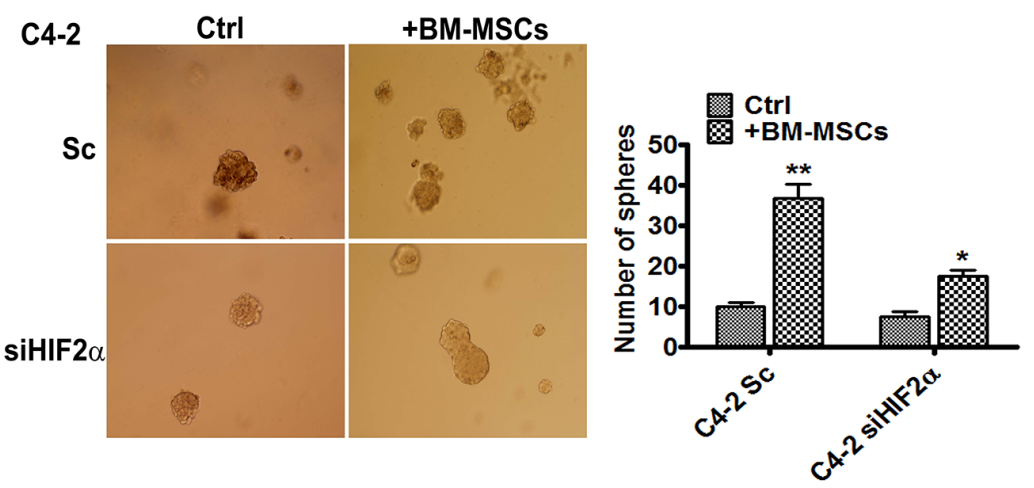

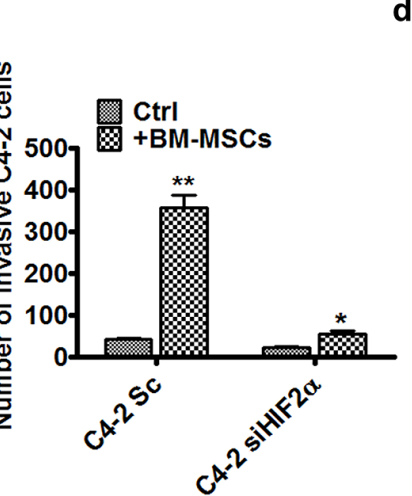

d.

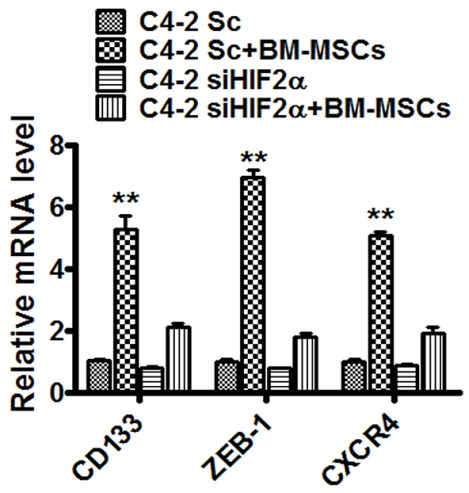

Figure 2: HIF2 $\alpha$ is essential for BM-MSCs increase of PCa invasion and stem cell population. a. qPCR analysis of expression of HIF2 $\alpha$ in C4-2 and CWR22RV1 (22RV1) cells after infection by the scramble (Sc) and siHIF2 $\alpha$ viruses. b. Sphere formation assay of the Sc or siHIF $2 \alpha$ C4-2 cells co-cultured with BM-MSCs (media as control). c. Invasion assay of the Sc or siHIF2 $\alpha$ C4-2 cells co-cultured with the BM-MSCs. d. qPCR analysis of expressions of the stem and metastasis marker genes. The Sc or siHIF2 $\alpha$ C4-2 cells were co-cultured with or without the primary BM-MSCs. The total RNAs were extracted and the expressions of the CD133, ZEB-1, and CXCR4 genes were analyzed.

after co-culture with BM-MSCs (Figure 4d), and addition of rCCL5 into PCa cells suppressed the PHD1 and PHD4 expressions at mRNA levels (Figure 4e).

Since the PHDs can influence the ubiquitination of HIF $2 \alpha$ by VHL E3-ligase [29], we further investigated whether the CCL5 mediated-HIF $2 \alpha$ upregulation is dependent on VHL. After knocking down the VHL in PCa cells by exploiting the VHLsiRNA, we observed that CCL5 no longer was able to mediate up-regulation of HIF $2 \alpha$ in C4-2 cells (Figure 4f). We also examined whether CCL5 could influence the interaction of HIF $2 \alpha$ and VHL molecules as early data showed that PHDs could hydroxylate the HIF2 $\alpha$ to promote its interaction with the VHL [28]. We performed a CoIP assay via co-transfection of the HA-tagged HIF $2 \alpha$ and GFP-VHL plasmids into the HEK293T cells in the absence and presence of rCCL5. After using the HA antibody to precipitate the HIF $2 \alpha$ molecule, the co-precipitated VHL protein with the GFP antibody was visualized by Western blot analysis. As shown in Figure $4 \mathrm{~g}$, we found the interaction between HIF $2 \alpha$ and VHL was significantly inhibited in the presence of $\mathrm{rCCL} 5$, suggesting that CCL5 suppressed the binding of HIF $2 \alpha$ and VHL to prevent degradation of the HIF $2 \alpha$ protein.

Together, results from Figure $4 \mathrm{a}-4 \mathrm{~g}$ suggest that CCL5 could regulate the ubquitination of HIF2 $\alpha$ via modulating the interaction between VHL and HIF $2 \alpha$.

\section{BM-MSCs and CCL5 induce HIF2 $\alpha$ to suppress $A R$ activity}

Early studies indicated that BM-MSCs and CCL5 could promote $\mathrm{PCa}$ invasion via regulating AR activity [11]. AR has been identified as a key factor to influence $\mathrm{PCa}$ progression [13, 30-32], and recent studies also documented well that AR might suppress $\mathrm{PCa}$ metastasis $[8,9,15,26,33]$. We were interested to see if CCL5-HIF2 $\alpha$ signals could function through altering the AR activity to increase the $\mathrm{PCa}$ cell invasion. We first knocked down the HIF $2 \alpha$ in C4-2 cells by HIF $2 \alpha$-siRNA, and then added rCCL5 to examine the expression of AR downstream genes including PSA and TMPRSS2. The results revealed that rCCL5 treatment 


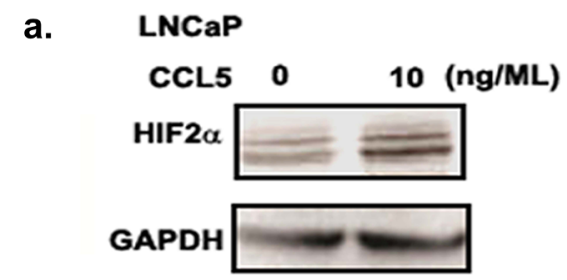

b.

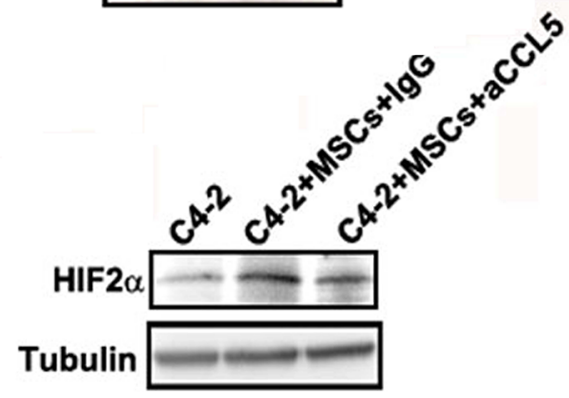

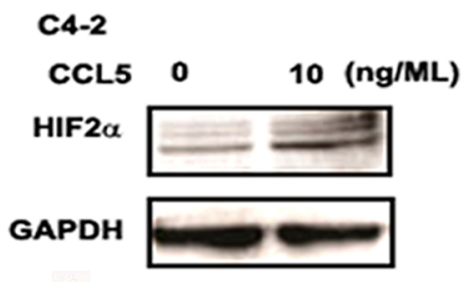

c.

d.
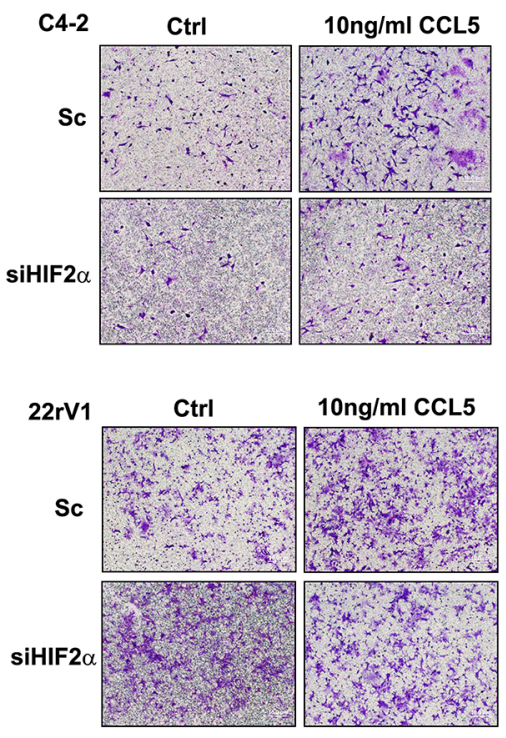
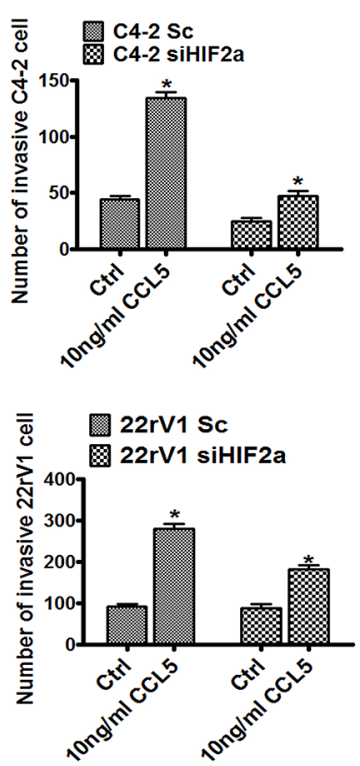

e.
22RV1

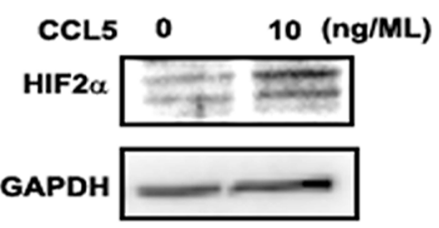

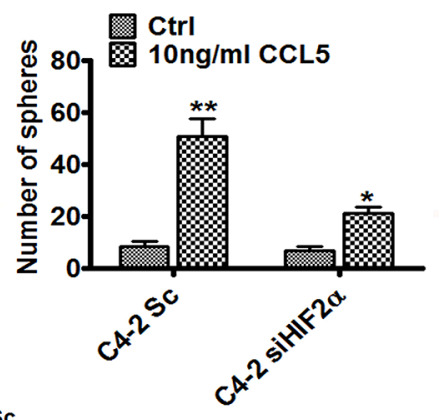
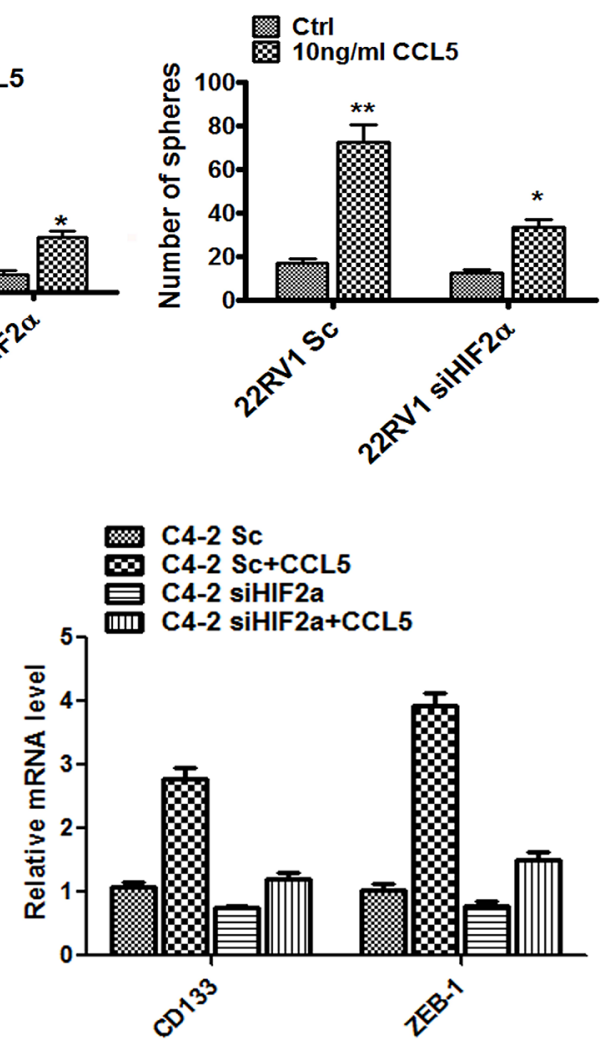

Figure 3: BM-MSCs secrete CCL5 to induce the HIF2 $\alpha$ expression. a. Western blot analysis results of HIF2 $\alpha$ expressions after CCL5 treatment. The PCa cells were treated with $10 \mathrm{ng} / \mathrm{ML}$ rCCL5 for 48 hrs and expressions of HIF2 $\alpha$ were analyzed. b. C4-2 cells were co-cultured with BM-MSCs (MSCs) and then treated with either IgG or CCL5 neutralizing antibody. The HIF2 $\alpha$ protein levels were then examined by Western blot analysis. $\mathbf{c}$. Sphere formation assay of Sc or siHIF2 $\alpha$ C4-2 and and CWR22RV1 (22RV1) cells treated by CCL5. The C4-2 and CWR22RV1 cells were infected by scramble or HIF2 $\alpha$ siRNA, and then cells were treated with $10 \mathrm{ng} / \mathrm{ml} \mathrm{CCL5}$ for 10 days. d. Invasion assay of C4-2 and CWR22RV1 cells treated with CCL5. The cells were treated with $10 \mathrm{ng} / \mathrm{ml}$ CCL5 for $72 \mathrm{hrs}$ and then the invasion assay was performed. e. The qPCR analysis of the mRNA level of CD133 and ZEB-1 in Sc/siHIF2 $\alpha$ C4-2 cells treated with $10 \mathrm{ng} / \mathrm{ml} \mathrm{CCL5.}$

suppressed the expression of PSA and TMPRSS2 in C4-2 cells, and knocking-down the HIF2 $\alpha$ interrupted the rCCL5 effect to suppress the expression of PSA and TMPRSS2 (Figure 5a). Knocking down HIF2 $\alpha$ in PCa cells also suppressed the BM-MSCs-induced PSA expression (Figure 5b), suggesting that CCL5 might be able to modulate HIF $2 \alpha$ signaling to influence AR activity. This conclusion was further demonstrated in the MMTV-ARE luciferase assay in HEK293T cells showing the addition of HIF $2 \alpha$ led to suppress MMTV-ARE luciferase activity at $1 \mathrm{nM}$ (human serum androgen concentration at castration stage) and $10 \mathrm{nM}$ DHT (normal human serum androgen concentration) conditions (Figure 5c).

To further dissect the mechanism how HIF $2 \alpha$ suppresses AR transactivation, we investigated whether HIF $2 \alpha$ could influence AR nuclear translocation, the key step to activate AR $[34,35]$, and results revealed that addition of HIF2 $\alpha$ suppressed the AR nuclear translocation (Figure 5d), suggesting that HIF $2 \alpha$ might be able to suppress the AR transactivation via inhibiting the AR translocation from cytosol into nucleus. 


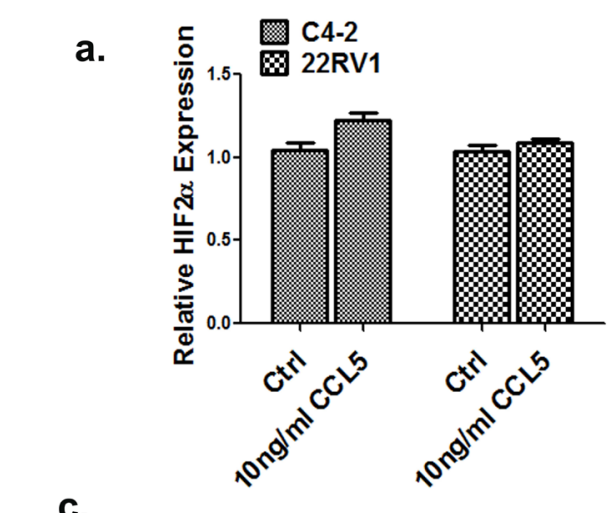

c.

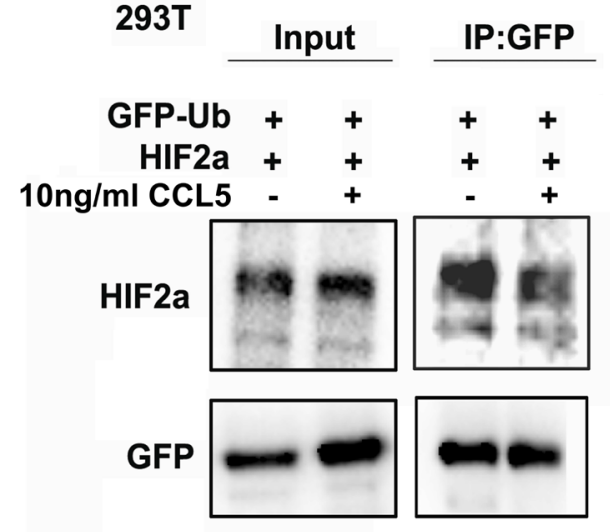

b.

$$
\text { C4-2 }
$$

DMSO

MG132

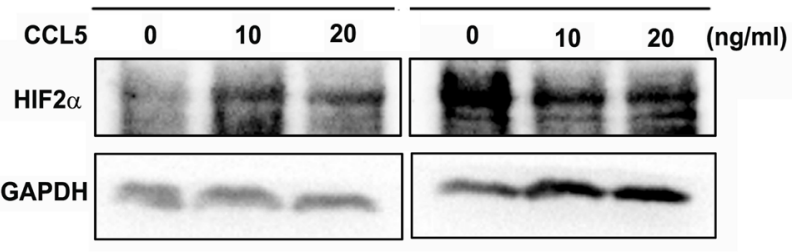

d.

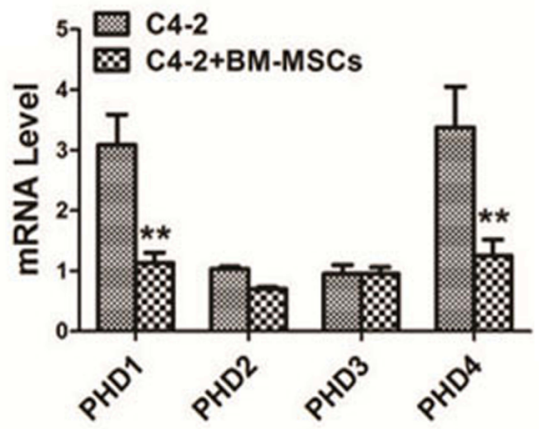

e.

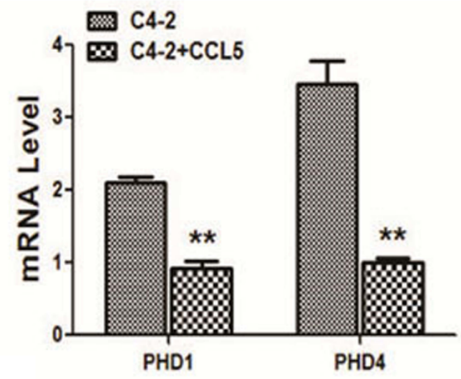

f.

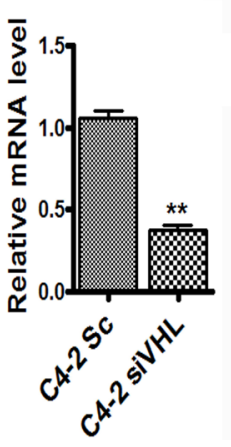

C4-2
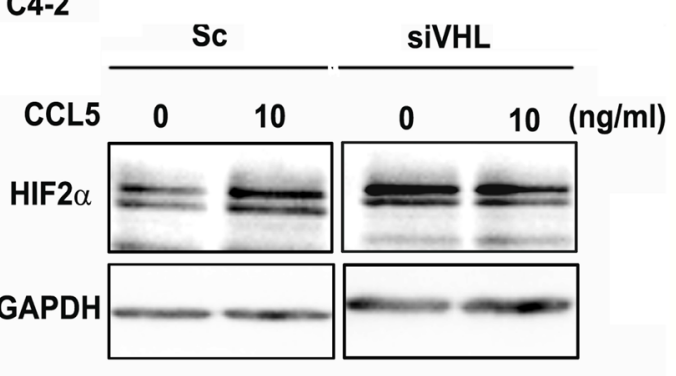

g.

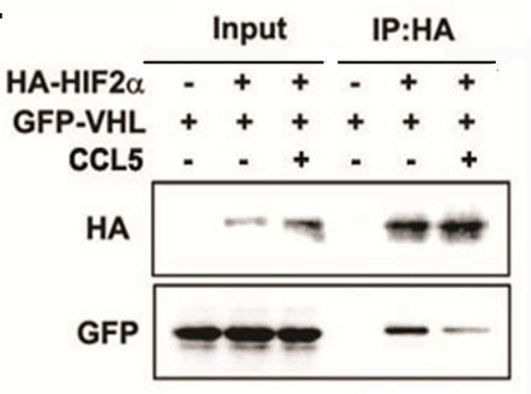

Figure 4: CCL5 suppresses the ubiquitination of HIF2 $\alpha$ through VHL. a. qPCR analysis of mRNA level of HIF2 $\alpha$ in PCa cells after treating with CCL5. b. The Western blot analysis of HIF2 $\alpha$ in C4-2 cells treated with different doses of CCL5 for 48 hrs, and then the DMSO or $20 \mu \mathrm{M} \mathrm{MG132}$ were added into the cells for $4 \mathrm{hrs}$. The expression of HIF $2 \alpha$ was analyzed. c. The ubiquitinaion assay of HIF2 $\alpha$ in 293 T cells. The 293 T cells were transfected with the GFP-Ub and HIF2 $\alpha$ plasmids. The GFP-Ub proteins were immuno-precipitated by GFP antibody, the HIF2 $\alpha$ proteins were analyzed by Western blot. d. The PHD1/PHD4 expression was regulated by BM-MSCs. The C2-4 cells were co-cultured with BM-MSCs and mRNA expression of PHDs was investigated by qPCR analysis. e. qPCR analysis of PHD1 and PHD4 after incubating C4-2 cells with $10 \mathrm{ng} / \mathrm{ml}$ rCCL5 for $48 \mathrm{hrs}$. f. The expression of HIF2 $\alpha$ in Sc/siVHL C4-2 cells after adding $10 \mathrm{ng} / \mathrm{ml} \mathrm{CCL5.}$ Left panel showed that the VHL was successfully knocked down in C4-2 cells. g. Co-IP experiment. 293 cells were transfected with HIF2 $\alpha$ and VHL, immunoprecipitated with HA antibody, and the VHL protein binding was detected in Western blot analysis using GFP antibody.

Since hypoxia can induce the expression of HIF $2 \alpha$, we next investigated whether hypoxia also can regulate the AR activity. The C4-2 and CWR22RV1 cells were cultured at $0.5 \%$ oxygen and expression of the AR downstream genes were analyzed by qPCR. Interestingly, the cells under hypoxia condition showed lower expressions of AR downstream genes, while also showing higher expression of the cancer stem cell also markers. (Figure 5e) This result is consistent with the previous reports suggesting that HIF $2 \alpha$ can increase cancer stem cell population and AR signaling is absent in cancer stem cells [26, 36].

Then we analyzed the AR localization under normoxia and hypoxia conditions. After culturing PCa cells under hypoxia condition for $24 \mathrm{hrs}$, we observed higher AR levels retained in the cytosol compared to the normoxia condition. (Figure 5f) The Western blot analysis also revealed that the 
a.

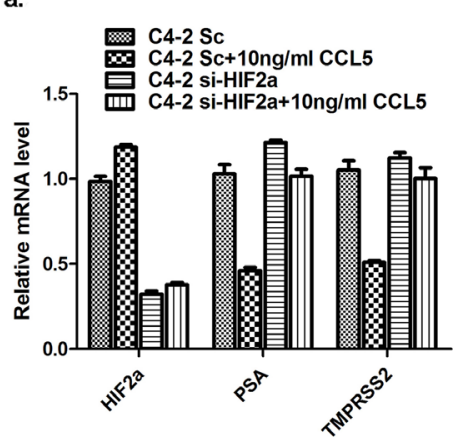

d.

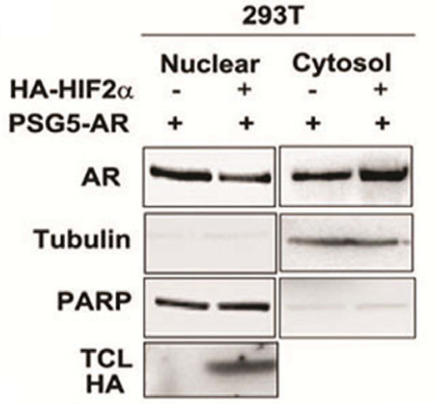

f.

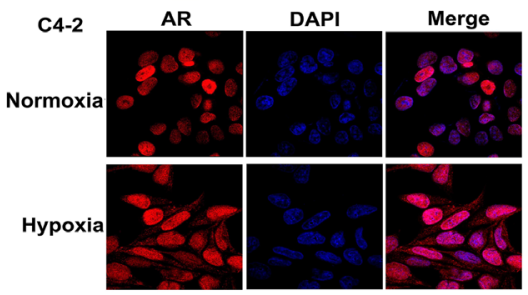

h.

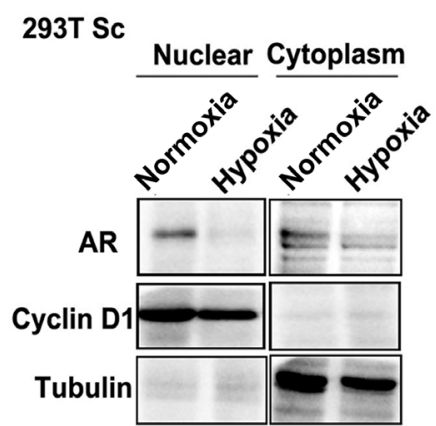

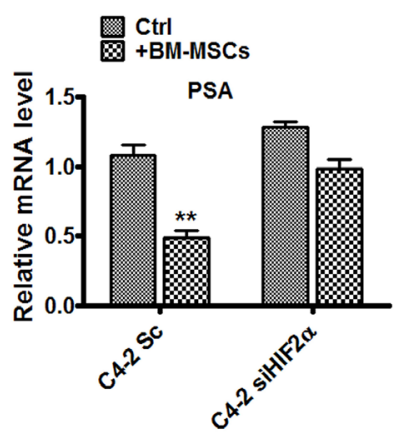

e.
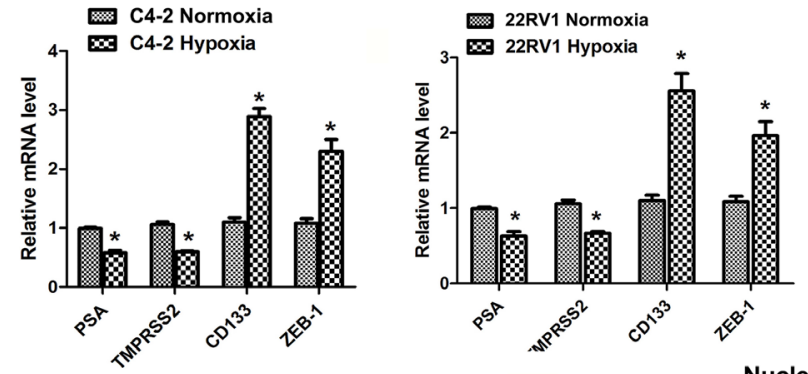

g.

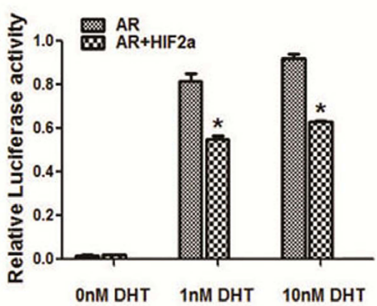

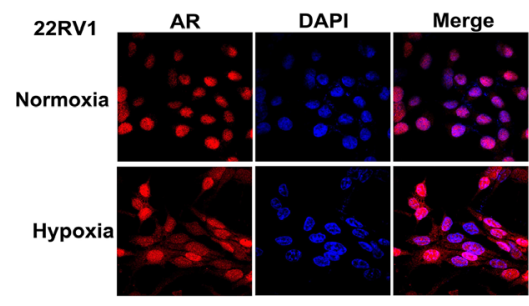

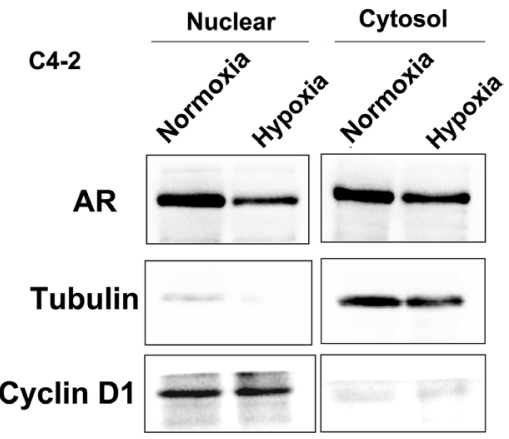

293T siHIF2a

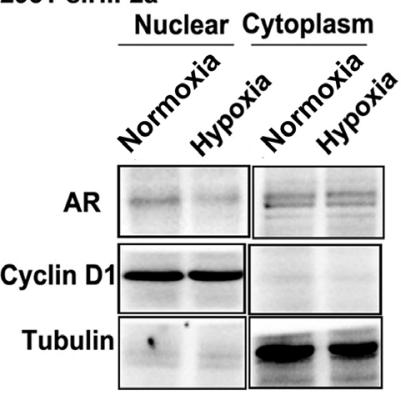

i.

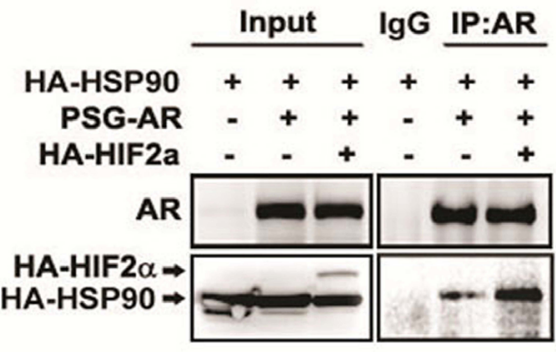

Figure 5: HIF2 $\alpha$ suppress AR transactivation activity. a. qPCR analysis of PSA, TMPRSS2 and HIF $2 \alpha$ expression after treating C4-2 cells with CCL5. b. Sc or siHIF2 $\alpha$ C4-2 cells were co-cultured with or without the BM-MSCs. The total RNAs were extracted and the expressions of the PSA genes were analyzed. c. HIF2 $\alpha$ regulates AR signal. The luciferase assay was performed using MMTV-luc in 293 cells in the absence and presence of HIF $2 \alpha$. d. HIF $2 \alpha$ regulates AR translocation. The 293 cells were transfected with the AR with or without HIF $2 \alpha$, the nuclear extraction were performed and detected by Western blot. Tubulin was used as the cytosol marker and the PARP was used as the nuclear marker. e. The qPCR analysis of PSA, TMPRSS2, CD133 and ZEB-1 expression in C4-2 and CWR22RV1 cells under normoxia and hypoxia $(0.5 \%$ oxygen) condition. $\mathbf{f}$. The immuno-fluorescent staining of AR under normoxia and hypoxia condition in C4-2 and CWR22RV1 cells. g. Hypoxia regulates the AR nuclear translocation. C4-2 cells were cultured under normoxia and hypoxia conditions, the nuclear extraction were performed and detected by Western blot. Tubulin was detected by Western blot as the cytosol marker; the cyclinD1 was detected as nuclear marker. h. Sc and siHIF2 $\alpha 293 \mathrm{~T}$ cells were cultured under normoxia or hypoxia condition. The AR localization was analyzed by western blot. i. The CoIP assay to detect the AR and HSP90 interaction was performed in 293 cells. Cells were transfected by HSP90, AR and HIF2 $\alpha$ plasmids. The AR antibody was used for the IP of AR-HSP90 interaction complex and the pull-down protein complex was further detected by western blot. 
AR translocation into the nuclear compartment in PCa cells was suppressed under hypoxia condition (Figure $5 \mathrm{~g}$ ).

We further knocked down the HIF2 $\alpha$ in $293 \mathrm{~T}$ cells, and treated cells with hypoxia. The results from Western blot analysis demonstrated that after knockingdown HIF $2 \alpha$, the hypoxia condition showed less effect to suppress the AR nuclear translocation. (Figure 5h), suggesting that HIF $2 \alpha$ is necessary for hypoxia inhibition of AR nuclear translocation.

As the heat shock protein 90 (HSP90) is an important factor that regulates AR nuclear translocation via binding to cytosol AR to influence its nuclear translocation [37], we also examined the potential influence of HIF $2 \alpha$ on the interaction of AR and HSP90. Using Co-IP assay, we found the interaction of these molecules was significantly increased upon HIF2 $\alpha$ addition (Figure 5i), suggesting that HIF $2 \alpha$ can suppress AR nuclear translocation via promoting the interaction of AR and HSP90 molecules.

Together, the results of Figure $5 \mathrm{a}-5 \mathrm{i}$ suggest that HIF $2 \alpha$ suppressed the activation of AR via blocking the AR nuclear translocation process.

\section{DISCUSSION}

Increasing numbers of reports suggested that BMMSCs might represent an important component in the TME to influence the tumor growth and metastasis $[4,5$,
38, 39]. An early study in breast cancer also suggested that CCL5 secreted from BM-MSCs might result in alteration of the cancer metastasis [38], yet the detailed mechanism involved in altering the downstream genes remained unclear. Here we report a new signaling from CCL5 secreted from BM-MSCs to increase the expression of HIF $2 \alpha$ in PCa that resulted in suppression of AR transactivation. The consequences of such suppression might then enhance the PCa invasion. The linkage of CCL5-HIF2 $\alpha$ signaling to AR-mediated PCa cell invasion described here might represent the first finding that provides us the opportunity to develop the potential new therapeutic approach to target this newly identified signaling to suppress the PCa metastasis (Figure 6).

The HIF family members are the key factors to respond to the hypoxia condition $[40,41]$. They can promote $\mathrm{PCa}$ cell growth, angiogenesis and metastasis. [42] There are two isoforms (HIF $1 \alpha$ and HIF2 $\alpha$ ) of the $\mathrm{HIF} \alpha$, and early studies were more focused on HIF $1 \alpha$ roles in PCa [43]. While we also found HIF1 $\alpha$ could be induced after recruited BM-MSCs (with increased CCL5) in PCa cells, we focused on HIF $2 \alpha$ here as the recent studies showed that the HIF $2 \alpha$ is more important for the cancer stem cell population induction $[24,25]$. The PDHs are the most important regulators for the HIF protein stability; they can catalyze the hydroxylation of the HIF $2 \alpha$ and then promote its

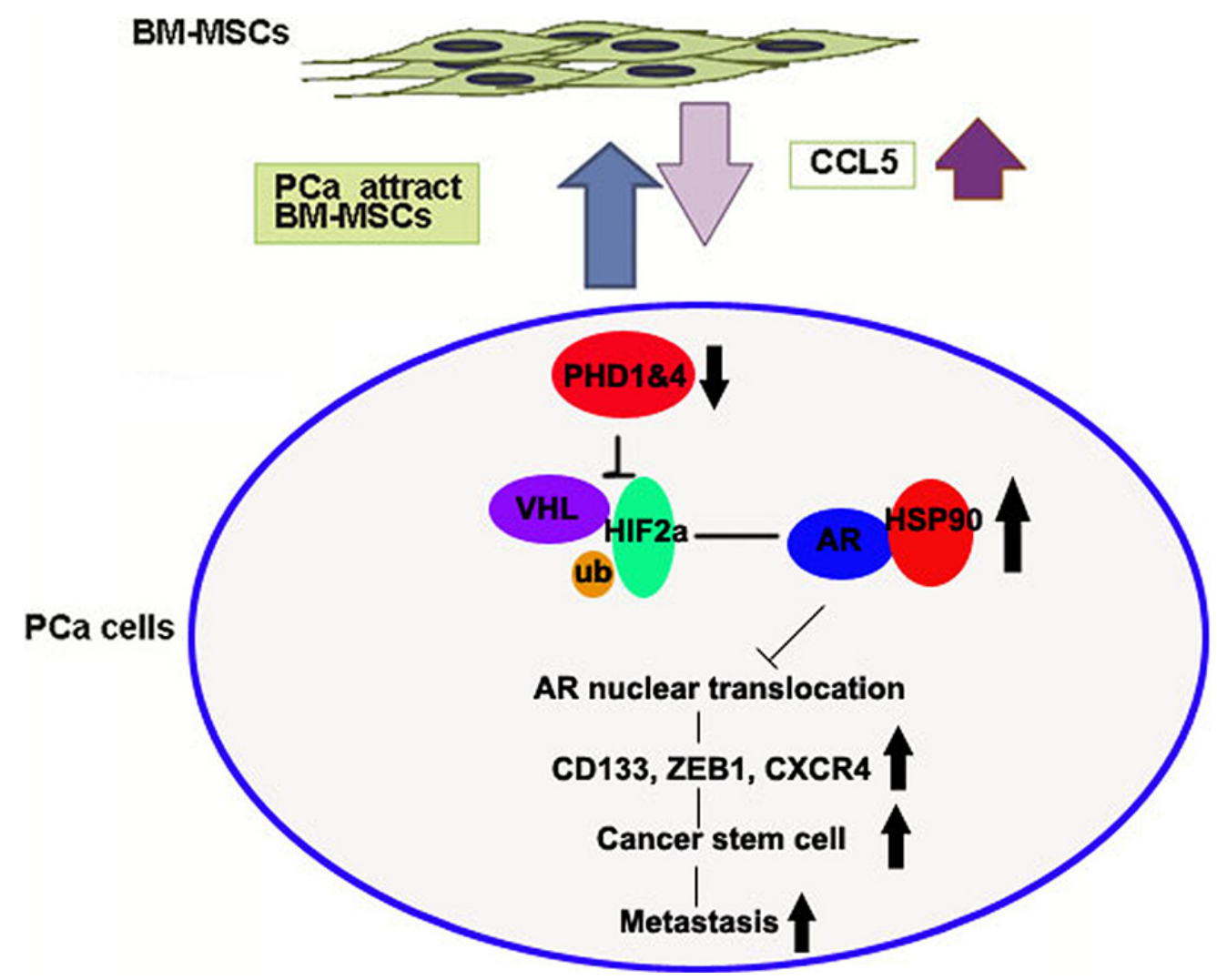

Figure 6: The cartoon of the pathway. 
binding to VHL, which is the E3-ligase of the HIF2 $\alpha$. These facts indicate that not only does low oxygen trigger the hypoxia system, but cytokines may also have the same effect.

While the AR has been extensively studied $[30-32,44]$ as a positive factor to promote PCa growth, and $\mathrm{ADT}$ to reduce or prevent androgens from binding to $\mathrm{AR}$ can effectively to suppress PCa cell growth during the first 12-24 months treatment, however, eventually most ADT fails and tumors re-grow with development of castration resistance [45-49]. However, more and more evidences with different approaches, suggested that $\mathrm{AR}$ might function as suppressor for the PCa metastasis. [9, 12, 13, 15, 26, 33]. Here we add evidence showing that infiltrating BM-MSCs can go through modulation of CCL5-HIF2 $\alpha$ signaling to suppress AR transactivation that may then enhance PCa cell invasion. Therefore, the development of a new combination therapy of ADT plus anti-CCL5/HIF $2 \alpha /$ AR signaling may help us to overcome the failure of ADT with better efficacy to suppress PCa cell invasion.

\section{MATERIALS AND METHODS}

\section{Cell culture}

LNCaP, C4-2, and CWR22RV1 cell lines were purchased from the American Type Culture Collection (ATCC, Manassas, VA) and cultured in RPMI 1640 with 10\% FBS. Human BM-MSCs were purchased from Stemcell Technologies (Vancouver, BC) and cultured in Human MesenCult ${ }^{\circledR}$ Proliferation Kit (Stemcell Technologies Inc). All cells were maintained in a humidified $5 \% \mathrm{CO}_{2}$ environment at $37^{\circ} \mathrm{C}$.

\section{Cell invasion assay}

Six $(0.4 \mu \mathrm{m}$ pore size $)$ or 24 -well $(8 \mu \mathrm{m}$ pore size $)$ transwell plates (Corning, Lowell, MA) were used for coculture and invasion assay, respectively. PCa cells were cocultured with BM-MSCs in transwell plates for 36-48 hrs. For in vitro invasion assays, transwell plate membranes were pre-coated with diluted matrigel (20\%) (BD Biosciences, Sparks, MD) and PCa cells ( $10^{5}$ cells in serum free medium) were plated in the upper chambers swhile 10\% serum containing media placed in the lower chambers. After 36-48 hrs incubation, cells invaded into the lower chambers and attached to the lower part of the membranes were stained with toluidine blue, and positively stained cells were counted. The cell numbers were counted in six random fields. Quantitation indicates means of triplicate repeats \pm SEM.

\section{RNA extraction and quantitative real-time PCR (qPCR) analysis}

Total RNAs were isolated using Trizol reagent (Invitrogen, Grand Island, NY). One $\mu \mathrm{g}$ of total RNA was subjected to reverse transcription using Superscript III transcriptase (Invitrogen, Grand Island, NY). qRT-PCR was conducted using a Bio-Rad CFX96 system with SYBR green to determine the mRNA expression level of a gene of interest. Expression levels were normalized to GAPDH level.

\section{Western blot analysis}

Cells were lysed in RIPA buffer and proteins (20-40 $\mu \mathrm{g}$ ) were separated on 8-10\% SDS/PAGE gel and then transferred onto PVDF membranes (Millipore, Billerica, MA). After blocking membranes, they were incubated with primary antibodies, HRP-conjugated secondary antibodies, and visualized using ECL system (Thermo Fisher Scientific, Rochester, NY). AR, GAPDH, tubulin, PARP, Cyclin D1 and GFP antibodies were from Santa Cruz Biotechnology, Inc (Santa Cruz, CA). HIF $2 \alpha$ antibody was purchased from Novus Biologicals (Littleton, CO) and Cell Signaling Technology (Beverly, MA)

\section{Histology and immunohistochemistry}

Mouse tissues obtained obtained were fixed in $10 \%(\mathrm{v} / \mathrm{v})$ formaldehyde in PBS, embedded in paraffin, and cut into 5- $\mu \mathrm{m}$ sections. Prostate tissue sections were deparaffinized in xylene solution and rehydrated and immunostaining was performed. HIF $2 \alpha$ antibody was purchased from Novus Biologicals (Littleton, CO).

\section{Luciferase assay}

PCa cells were plated in 24-well plates and transfected with MMTV-luc containing ARE sequence using Lipofectamine (Invitrogen, Grand Island, NY). After transfection, regular media were added with various DHT concentrations, 0 (ethanol as vehicle control), $1 \mathrm{nM}$, and $10 \mathrm{nM}$, and incubated for $48 \mathrm{hrs}$. pRL-TK was used as internal control. Luciferase activity was measured by Dual-Luciferase Assay (Promega, Madison, WI) according to the manufacturer's manual.

\section{In vivo orthotopic mice injection}

The nude mice were from the Jackson Lab (Bar Harbor, Maine). CWR22RV1 cells were engineered to express luciferase reporter gene (PCDNA3.0-luciferase) by stable transfection and the positive stable clones (luc-CWR22RV1) were selected and expanded in culture. 20 control group mice (6-8 wks) were injected with luc-CWR22RV1 cells $\left(1 \times 10^{6}\right.$, mixed with Matrigel, 1:1) and 10 test group mice were co-injected with PCa cells with primary BM-MSCs $\left(1 \times 10^{5}\right)$. After sacrificing mice at $6 \mathrm{wks}$, the tumors were analyzed by IHC staining. 


\section{Statistics}

The data values were presented as the mean \pm SEM. Differences in mean values between two groups were analyzed by two-tailed Student's $t$ test. $p \leq 0.05$ was considered statistically significant. *, $P$ value $<0.05$; **, $P$ value $<0.005$.

\section{CONCLUSION}

Together, our studies suggest that recruited BMMSCs can secrete CCL5 to suppress the PHDs expression to induce the HIF $2 \alpha$ expression. The consequences of such induced HIF2 $\alpha$ expression can lead to enhance the AR-HSP90 interaction to suppress the AR nuclear translocation and $\mathrm{AR}$ transactivation that results in promotion of the PCa metastasis.

\section{ACKNOWLEDGMENTS}

We thank Karen Wolf for help with manuscript preparation. This work was supported by NIH grants (CA122840 and CA256700), Taiwan Department of Health Clinical Trial and Research Center of Excellence (DOH102-TD-B-111-004) to China Medical University, Taiwan; and National Basic Research Program of China (973 Program), Grant Numbers: 2012CB518304.

\section{CONFLICTS OF INTEREST}

The authors have no conflicts of interests to declare.

\section{REFERENCES}

1. Zhu P, Baek S.H, Bourk E.M, Ohgi K.A, Garcia-Bassets I, Sanjo H, Akira S, Kotol P.F, Glass C.K, Rosenfeld M.G, et al. Macrophage/cancer cell interactions mediate hormone resistance by a nuclear receptor derepression pathway. Cell. 2006; 124:615-629.

2. Zeng Y, Opeskin K, Goad J, Williams E.D. Tumor-induced activation of lymphatic endothelial cells via vascular endothelial growth factor receptor-2 is critical for prostate cancer lymphatic metastasis. Cancer Res. 2006; 66:9566-9575.

3. Augsten M, Hagglof C, Olsson E, Stolz C, Tsagozis P, Levchenko T, Frederick M.J, Borg A, Micke P, Egevad L, et al. CXCL14 is an autocrine growth factor for fibroblasts and acts as a multi-modal stimulator of prostate tumor growth. Proc Natl Acad Sci U S A. 2009; 106:3414-3419.

4. Quante M, Tu S.P, Tomita H, Gonda T, Wang S.S, Takashi S, Baik G.H, Shibata W, Diprete B, Betz K.S, et al. Bone marrow-derived myofibroblasts contribute to the mesenchymal stem cell niche and promote tumor growth. Cancer Cell. 2011; 19:257-272.

5. Kobayashi A, Okuda H, Xing F, Pandey P.R, Watabe M, Hirota S, Pai S.K, Liu W, Fukuda K, Chambers C, et al.
Bone morphogenetic protein 7 in dormancy and metastasis of prostate cancer stem-like cells in bone. J Exp Med. 2011; 208:2641-2655.

6. Ammirante M, Luo J.L, Grivennikov S, Nedospasov S, Karin M. B-cell-derived lymphotoxin promotes castrationresistant prostate cancer. Nature. 2010; 464:302-305.

7. Fang L.Y, Izumi K, Lai K.P, Liang L, Li L, Miyamoto H, Lin W.J, Chang C. Infiltrating macrophages promote prostate tumorigenesis via modulating androgen receptor-mediated CCL4-STAT3 signaling. Cancer Res. 2013; 73:5633-5646.

8. Izumi K, Mizokami A, Lin W.J, Lai K.P, Chang C. Androgen receptor roles in the development of benign prostate hyperplasia. Am J Pathol. 2013; 182:1942-1949.

9. Lin T.H, Izumi K, Lee S.O, Lin W.J, Yeh S, Chang C. Anti-androgen receptor ASC-J9 versus anti-androgens MDV3100 (Enzalutamide) or Casodex (Bicalutamide) leads to opposite effects on prostate cancer metastasis via differential modulation of macrophage infiltration and STAT3CCL2 signaling. Cell Death Dis. 2013; 4:e764.

10. Wang X, Lee S.O, Xia S, Jiang Q, Luo J, Li L, Yeh S, Chang C. Endothelial cells enhance prostate cancer metastasis via IL-6 $\longrightarrow$ androgen receptor $\longrightarrow$ TGF-beta $\longrightarrow$ MMP-9 signals. Mol Cancer Ther. 2013; 12:1026-1037.

11. Luo J, Ok Lee S, Liang L, Huang C.K, Li L, Wen S, Chang C. Infiltrating bone marrow mesenchymal stem cells increase prostate cancer stem cell population and metastatic ability via secreting cytokines to suppress androgen receptor signaling. Oncogene. 2013.

12. Niu Y, Altuwaijri S, Lai K.P, Wu C.T, Ricke W.A, Messing E.M, Yao J, Yeh S, Chang C. Androgen receptor is a tumor suppressor and proliferator in prostate cancer. Proc Natl Acad Sci U S A. 2008; 105:12182-12187.

13. Niu Y, Chang T.M, Yeh S, Ma W.L, Wang Y.Z, Chang C. Differential androgen receptor signals in different cells explain why androgen-deprivation therapy of prostate cancer fails. Oncogene. 2010; 29:3593-3604.

14. Carter H.B. American Urological Association (AUA) Guideline on prostate cancer detection: process and rationale. BJU Int. 2013; 112:543-547.

15. Lin T.H, Lee S.O, Niu Y, Xu D, Liang L, Li L, Yeh S.D, Fujimoto N, Yeh S, Chang C. Differential Androgen Deprivation Therapies with Anti-androgens Casodex/ Bicalutamide or MDV3100/Enzalutamide versus Antiandrogen Receptor ASC-J9(R) Lead to Promotion versus Suppression of Prostate Cancer Metastasis. J Biol Chem. 2013; 288:19359-19369.

16. Poon E, Harris A.L, Ashcroft M. Targeting the hypoxiainducible factor (HIF) pathway in cancer. Expert Rev Mol Med. 2009; 11:e26.

17. Haddad N.M, Cavallerano J.D, Silva P.S. Von hippel-lindau disease: a genetic and clinical review. Semin Ophthalmol. 2013; 28:377-386.

18. Semenza G.L. Hypoxia-inducible factors: mediators of cancer progression and targets for cancer therapy. Trends Pharmacol Sci. 2012; 33:207-214. 
19. Brahimi-Horn M.C, Pouyssegur J. HIF at a glance. J Cell Sci. 2009; 122:1055-1057.

20. Huang L.E, Bunn H.F. Hypoxia-inducible factor and its biomedical relevance. J Biol Chem. 2003; 278:19575-19578.

21. Greijer A.E, van der Wall E. The role of hypoxia inducible factor 1 (HIF-1) in hypoxia induced apoptosis. J Clin Pathol. 2004; 57:1009-1014.

22. Pastrana E, Silva-Vargas V, Doetsch F. Eyes wide open: a critical review of sphere-formation as an assay for stem cells. Cell Stem Cell. 2011; 8:486-498.

23. Kleinman, H.K., Jacob, K. Invasion assays. Curr Protoc Cell Biol Chapter. 2001; 12:Unit 1212.

24. Koh M.Y, Lemos R Jr, Liu X, Powis G. The hypoxiaassociated factor switches cells from HIF-1alpha- to HIF2alpha-dependent signaling promoting stem cell characteristics, aggressive tumor growth and invasion. Cancer Res. 2011; 71:4015-4027.

25. Mimeault M, Batra S.K. Hypoxia-inducing factors as master regulators of stemness properties and altered metabolism of cancer- and metastasis-initiating cells. J Cell Mol Med. 2013; 17:30-54.

26. Lee S.O, Ma Z, Yeh C.R, Luo J, Lin T.H, Lai K.P, Yamashita S, Liang L, Tian J, Li L, et al. New therapy targeting differential androgen receptor signaling in prostate cancer stem/progenitor vs. non-stem/progenitor cells. J Mol Cell Biol. 2013; 5:14-26.

27. Sen Banerjee S, Thirunavukkarasu M, Tipu Rishi M, Sanchez J.A, Maulik N, Maulik G. HIF-prolyl hydroxylases and cardiovascular diseases. Toxicol Mech Methods. 2012; 22:347-358.

28. Rankin E.B, Biju M.P, Liu Q, Unger T.L, Rha J, Johnson R.S, Simon M.C, Keith B, Haase V.H. Hypoxiainducible factor-2 (HIF-2) regulates hepatic erythropoietin in vivo. J Clin Invest. 2007; 117:1068-1077.

29. Maxwell P.H, Wiesener M.S, Chang G.W, Clifford S.C, Vaux E.C, Cockman M.E, Wykoff C.C, Pugh C.W, Maher E.R, Ratcliffe P.J. The tumour suppressor protein VHL targets hypoxia-inducible factors for oxygendependent proteolysis. Nature. 1999; 399:271-275.

30. Chang C.S, Kokontis J, Liao S.T. Molecular cloning of human and rat complementary DNA encoding androgen receptors. Science. 1988; 240:324-326.

31. Heinlein C.A, Chang C. Androgen receptor in prostate cancer. Endocr Rev. 2004; 25:276-308.

32. Wen S, Niu Y, Lee S.O, Chang C. Androgen Receptor (AR) Positive vs Negative Roles in Prostate Cancer Cell Deaths including Apoptosis, Anoikis, Entosis, Necrosis and Autophagic Cell Death. Cancer Treatment Reviews 2014; 40:31-40.

33. Niu Y, Altuwaijri S, Yeh S, Lai K.P, Yu S, Chuang K.H, Huang S.P, Lardy H, Chang C. Targeting the stromal androgen receptor in primary prostate tumors at earlier stages. Proc Natl Acad Sci U S A. 2008; 105:12188-12193.

34. Lai K.P, Huang C.K, Chang Y.J, Chung C.Y, Yamashita S, Li L, Lee S.O, Yeh S, Chang C. New therapeutic approach to suppress castration-resistant prostate cancer using ASCJ9 via targeting androgen receptor in selective prostate cells. Am J Pathol. 2013; 182:460-473.

35. Yamashita S, Lai K.P, Chuang K.L, Xu D, Miyamoto H, Tochigi T, Pang S.T, Li L, Arai Y, Kung H.J, et al. ASC-J9 suppresses castration-resistant prostate cancer growth through degradation of full-length and splice variant androgen receptors. Neoplasia. 2012; 14:74-83.

36. Ma Y, Liang D, Liu J, Axcrona K, Kvalheim G, Stokke T, Nesland J.M, Suo Z. Prostate cancer cell lines under hypoxia exhibit greater stem-like properties. PLoS One. 2011; 6:e29170.

37. Fang Y, Fliss A.E, Robins D.M, Caplan A.J. Hsp90 regulates androgen receptor hormone binding affinity in vivo. J Biol Chem. 1996; 271:28697-28702.

38. Karnoub A.E, Dash A.B, Vo A.P, Sullivan A, Brooks M.W, Bell G.W, Richardson A.L, Polyak K, Tubo R, Weinberg R.A. Mesenchymal stem cells within tumour stroma promote breast cancer metastasis. Nature. 2007; 449:557-563.

39. Ye H, Cheng J, Tang Y, Liu Z, Xu C, Liu Y, Sun Y. Human bone marrow-derived mesenchymal stem cells produced TGFbeta contributes to progression and metastasis of prostate cancer. Cancer Invest. 2012; 30:513-518.

40. Maher E.R. HIF2 and endocrine neoplasia: an evolving story. Endocr Relat Cancer. 2013; 20:C5-7.

41. Pawlus M.R, Hu C.J. Enhanceosomes as integrators of hypoxia inducible factor (HIF) and other transcription factors in the hypoxic transcriptional response. Cell Signal. 2013; 25:1895-1903.

42. Kimbro K.S, Simons J.W. Hypoxia-inducible factor-1 in human breast and prostate cancer. Endocr Relat Cancer. 2006; 13:739-749.

43. Amankwah E.K, Sellers T.A, Park J.Y. Gene variants in the angiogenesis pathway and prostate cancer. Carcinogenesis. 2012; 33:1259-1269.

44. Heinlein C.A, Chang C. Androgen receptor (AR) coregulators: an overview. Endocr Rev. 2002; 23:175-200.

45. Hammerer P, Madersbacher S. Landmarks in hormonal therapy for prostate cancer. BJU Int. 2012; 110:23-29.

46. Tsao C.K, Galsky M.D, Small A.C, Yee T, Oh W.K. Targeting the androgen receptor signalling axis in castration-resistant prostate cancer (CRPC). BJU Int. 2012; 110:1580-1588.

47. Miyamoto H, Messing E.M, Chang C. Androgen deprivation therapy for prostate cancer: current status and future prospects. Prostate. 2004; 61:332-353.

48. Miyamoto H, Messing E.M, Chang C. Does androgen deprivation improve treatment outcomes in patients with low-risk and intermediate-risk prostate cancer? Nat Clin Pract Oncol. 2005; 2:236-237.

49. Miyamoto H, Altuwaijri S, Cai Y, Messing E.M, Chang C. Inhibition of the Akt, cyclooxygenase-2, and matrix metalloproteinase- 9 pathways in combination with androgen deprivation therapy: potential therapeutic approaches for prostate cancer. Mol Carcinog. 2005; 44:1-10. 